\title{
RATIONAL GROUP ACTIONS ON AFFINE PI-ALGEBRAS
}

\author{
MARTIN LORENZ \\ Department of Mathematics, Temple University, Philadelphia, PA 19122, USA \\ e-mail:lorenz@temple.edu \\ To Kenny Brown and Toby Stafford on the occasion of their 60th birthdays
}

\begin{abstract}
Let $R$ be an affine PI-algebra over an algebraically closed field $\mathbb{k}$ and let $G$ be an affine algebraic $\mathbb{k}$-group that acts rationally by algebra automorphisms on $R$. For $R$ prime and $G$ a torus, we show that $R$ has only finitely many $G$-prime ideals if and only if the action of $G$ on the centre of $R$ is multiplicity free. This extends a standard result on affine algebraic $G$-varieties. Under suitable hypotheses on $R$ and $G$, we also prove a PI-version of a well-known result on spherical varieties and a version of Schelter's catenarity theorem for $G$-primes.
\end{abstract}

2000 Mathematics Subject Classification. 16R30, 16W22.

\section{Introduction.}

1.1. This paper addresses the following general question:

Suppose a group $G$ acts by automorphisms on a ring $R$. When does $R$ have only finitely many $G$-prime ideals?

Recall that a proper $G$-stable (two-sided) ideal $I$ of $R$ is called $G$-prime if $A B \subseteq I$ for $G$-stable ideals $A$ and $B$ of $R$ implies that $A \subseteq I$ or $B \subseteq I$. The set of all $G$-prime ideals of $R$ will be denoted by

\section{$G-\operatorname{Spec} R$.}

Continuing our investigations in [21] and [22], our main focus will be on the case where $R$ is an algebra over an algebraically closed base field $\mathbb{k}$ and $G$ is an affine algebraic $\mathbb{k}$-group that acts rationally by $\mathbb{k}$-algebra automorphisms on $R$; see 2.2 below for a brief reminder on rational actions. This setting will be assumed for the remainder of the Introduction. For noetherian $R$ and an algebraic torus $G$, the above question was stated as Problem II.10.6 in [7].

1.2 The question in Section 1 is motivated in part by the stratification of the prime spectrum $\operatorname{Spec} R$ that is induced by the action of $G$. Namely, there is a surjection

$$
\text { Spec } R \rightarrow G-\operatorname{Spec} R
$$

sending a given prime ideal $P$ to the largest $G$-stable ideal of $R$ that is contained in $P$. A precise description of the fibres of this map in terms of commutative algebras is given in [22, Theorem 9]. Hence, from a noncommutative perspective, the focus shifts 
to the description of $G$-Spec $R$, with finiteness being the optimal scenario. It turns out that, as long as the deformation parameters are sufficiently generic, $G$-Spec $R$ is indeed finite for all quantized coordinate algebras $R=\mathcal{O}_{\mathbf{q}}(X)$ that have been analysed in detail thus far, the acting group $G$ typically being a suitably chosen algebraic torus. Notable examples include the (generic) quantized coordinate rings of all semisimple algebraic groups (Joseph [14], Hodges, Levasseur and Toro [12]), quantum matrices and quantum Grassmannians (Cauchon, Lenagan and others; e.g, [8], [9] and [19]). Finiteness of $G$-Spec $R$ has also been observed for Leavitt path algebras $R$, again for the action of a suitable torus $G$ [1]. These finiteness results depend either on long calculations in $R$ or else on finding a presentation of $R$ as an iterated skew polynomial algebra, a class of algebras for which a finiteness result due to Goodearl and Letzter $[10,11]$ is available. A general finiteness criterion for $G$-Spec $R$ is currently lacking.

1.3. In this paper, we concentrate on the case of an affine $\mathbb{k}$-algebra $R$ satisfying a polynomial identity (PI). The class of PI-algebras, whose structural theory was pioneered by Kaplansky [15], Amitsur [2] and Procesi [27], combines aspects of noncommutativity with geometric properties that are familiar from commutative algebras. In order to give the finiteness problem in Section 1 a geometric perspective, we mention the following connection with $G$-orbits of rational ideals. Here, a prime ideal $P$ of $R$ is called rational if $\mathcal{C}(R / P)=\mathbb{k}$, where $\mathcal{C}(\cdot)$ denotes the centre of the classical ring of quotients of the ring in question. Rational primes are exactly the closed points of Spec $R$; see Section 2.3.4 below for several equivalent characterizations of rationality. An ideal $P \in G$-Spec $R$ is said to be $G$-rational if the algebra of $G$-invariants $\mathcal{C}(R / P)^{G}$ coincides with $\mathbb{k}$. The subset of $\operatorname{Spec} R$ consisting of all rational primes of $R$ will be denoted by $\operatorname{Rat} R$, and $G$-Rat $R \subseteq G$-Spec $R$ will denote the set of all $G$-rational ideals. Since $R$ satisfies the ascending chain condition for semiprime ideals (2.3.1), the Nullstellensatz (2.3.3) and the Dixmier-Mœglin equivalence (2.3.4), the following result is a special case of [22, Proposition 14].

PROPOSITION 1. Let $R$ be an affine PI-algebra over the algebraically closed field $\mathbb{k}$ and let $G$ be an affine algebraic $\mathbb{k}$-group that acts rationally by $\mathbb{k}$-algebra automorphisms on $R$. Then the following are equivalent:

(i) $G$-Spec $R$ is finite;

(ii) $G$-Rat $R$ is finite;

(iii) $G$ has finitely many orbits in Rat $R$;

(iv) $G$-Rat $R=G$-Spec $R$.

Thus, the finiteness problem at hand amounts to determining when all $G$-primes of $R$ are $G$-rational.

1.4. In studying the finiteness question in Section 1 we may assume without loss that $G$ is connected. In this case, all $G$-primes of $R$ are actually prime, and hence $G$-Spec $R$ is the set of all $G$-stable prime ideals of $R$; see Lemma 4 below. The main result of this note concerns the special case where $R$ is an affine PI-algebra and $G$ is a torus; it generalizes a standard result on affine algebraic $G$-varieties [17, II.3.3 Satz 5].

THEOREM 2. Let $R$ be a prime affine PI-algebra over the algebraically closed field $\mathbb{k}$ and let $G$ be an algebraic $\mathbb{k}$-torus that acts rationally by $\mathbb{k}$-algebra automorphisms on $R$. Then $G-\operatorname{Spec} R$ is finite if and only if the action of $G$ on the centre $\mathcal{Z}(R)$ is multiplicity 
free: for each rational character $\lambda: G \rightarrow \mathbb{k}^{\times}$, the weight space $\mathcal{Z}(R)_{\lambda}=\{r \in \mathcal{Z}(R) \mid g . r=$ $\lambda(\mathrm{g}) \mathrm{r}$ for all $\mathrm{g} \in \mathrm{G}\}$ has dimension at most 1 .

The proof of Theorem 2 will be given in Section 3 after deploying some auxiliary results and a generous amount of background material in Section 2. We remark that if $R$ is also assumed noetherian, then Theorem 2 is quite a bit easier, being an immediate consequence of Proposition 7 and Lemma 8(b) below. We conclude, in Section 4, with two results for noetherian $R$, namely a PI-version of a standard result on spherical varieties (Proposition 10) and a version of Schelter's catenarity theorem for $G$-primes (Proposition 11).

NotATIONS AND CONVENTIONS. All rings have a 1 which is inherited by subrings and preserved under homorphisms. The action of the group $G$ on the $\operatorname{ring} R$ will be written as $G \times R \rightarrow R,(g, r) \mapsto g . r$. For any ideal $I$ of $R$, we will write $I: G=\bigcap_{g \in G} g . I$; this is the largest $G$-stable ideal of $R$ that is contained in $I$. The symbol $\subset$ denotes a proper inclusion.

\section{Preliminaries.}

2.1. Finite centralizing ring extensions. A ring extension $R \subseteq S$ is called centralizing if $S=R \mathbf{C}_{S}(R)$, where $\mathbf{C}_{S}(R)=\{s \in S \mid s r=r s$ for all $r \in R\}$. In this case, for any prime ideal $P$ of $S$, the contraction $P \cap R$ is easily seen to be a prime ideal of $R$. A centralizing extension $R \subseteq S$ is called finite, if $S$ is finitely generated as left or, equivalently, right $R$-module. By results of Bergman [3, 4] (see also [29]), the classical relations of lying over and incomparability for prime ideals hold in any finite centralizing extension $R \subseteq S$ :

- given $Q \in \operatorname{Spec} R$, there exists $P \in \operatorname{Spec} S$ such that $Q=P \cap R \quad$ (Lying Over);

- if $P, P^{\prime} \in \operatorname{Spec} S$ are such that $P \subset P^{\prime}$ then $P \cap R \subset P^{\prime} \cap R \quad$ (Incomparability).

LeMmA 3. Let $R \subseteq S$ be a finite centralizing extension of rings and let $G$ be a group acting by automorphisms on $S$ that stabilize $R$. Assume that every ideal $A$ of $S$ contains a finite product of primes each of which contains $A$. Then contraction yields a surjective map

$$
G \text {-Spec } S \rightarrow G \text {-Spec } R, \quad I \mapsto I \cap R
$$

with finite fibres. In particular, if one of $G-\operatorname{Spec} S$ or $G$-Spec $R$ is finite then so is the other.

Proof. First, we note that the $G$-primes of $S$ are exactly the ideals of the form $P: G$ with $P \in \operatorname{Spec} S$. Indeed, it is straightforward to check that $P: G$ is $G$-prime. Conversely, for any given $I \in G$-Spec $S$, there are finitely many $P_{i} \in \operatorname{Spec} S$ (not necessarily distinct) with $I \subseteq P_{i}$ and $\prod_{i} P_{i} \subseteq I$. But then $I \subseteq P_{i}: G$ for each $i$ and $\prod_{i} P_{i}: G \subseteq I$, whence $I=$ $P_{i}$ : $G$ for some $i$. In particular, each $I \in G$-Spec $S$ is semiprime. The group $G$ permutes the finitely many primes of $S$ that are minimal over $I$ and $G$-primeness forces these primes to form a single $G$-orbit. Therefore, we may write $I=P: G$ with $P \in \operatorname{Spec} S$ having a finite $G$-orbit. Similar remarks apply to the ring $R$, because every ideal $B$ of $R$ also contains a finite product of primes each of which contains $B$; this follows from the fact that $B$ contains some finite power of $B S \cap R$ by [20, Corollary 1.4].

Now let $I \in G$-Spec $S$ be given and let $A, B$ be $G$-stable ideals of $R$ such that $A B \subseteq$ $I \cap R$. Then, $A S=S A$ is a $G$-stable ideal of $S$ and similarly for $B$. Since $(A S)(B S)=$ 
$A B S \subseteq I$, we must have $A S \subseteq I$ or $B S \subseteq I$ and hence $A \subseteq I \cap R$ or $B \subseteq I \cap R$. Thus, contraction yields a well-defined map $G$-Spec $S \rightarrow G$-Spec $R$.

For surjectivity of the contraction map, let $J \in G$-Spec $R$ be given and write $J=Q: G$ with $Q \in \operatorname{Spec} R$. By Lying Over we may choose $P \in \operatorname{Spec} S$ with $Q=P \cap R$. Putting $I=P: G$ we obtain a $G$-prime of $S$ such that $J=I \cap R$.

Finally, assume that $I \in G$-Spec $S$ contracts to a given $J \in G$-Spec $R$. Write $I=P: G$ with $P \in \operatorname{Spec} S$ having a finite $G$-orbit. We claim that $P$ must be minimal over the ideal $J S$. Indeed, suppose that $J S \subseteq P^{\prime} \subset P$ for some $P^{\prime} \in \operatorname{Spec} S$. Then Incomparability gives $P \cap R \supset P^{\prime} \cap R \supseteq J=\bigcap_{g \in G} g .(P \cap R)$. Since the last intersection is finite and $P^{\prime} \cap R$ is prime, we conclude that $g .(P \cap R) \subseteq P^{\prime} \cap R$ for some $g \in G$. Consequently, g. $(P \cap R) \subset P \cap R$, which is impossible. This proves minimality of $P$ over $J S$. It follows that there are finitely many possibilities for $P$, and hence there are finitely many possibilities for $I$. This completes the proof of the lemma.

The hypothesis that every ideal of $S$ contains a finite product of prime divisors is of course satisfied, by Noether's classical argument, if $S$ satisfies the ascending chain condition for ideals. More importantly, for our purposes, the hypothesis also holds for any affine PI-algebra $S$ over some commutative noetherian ring by Braun's theorem $[30,6.3 .39]$.

2.2. Rational group actions. Let $G$ be an affine algebraic $\mathbb{k}$-group, where $\mathbb{k}$ is an algebraically closed field, and let $\mathbb{k}[G]$ denote the Hopf algebra of regular functions on $G$. A $\mathbb{k}$-vector space $M$ is called a $G$-module if $M$ is a $\mathbb{k}[G]$-comodule; see Jantzen [13, 2.7-2.8] or Waterhouse [32, 3.1-3.2]. Writing the comodule structure map $\Delta_{M}: M \rightarrow$ $M \otimes \mathbb{k}[G]$ as $\Delta_{M}(m)=\sum m_{0} \otimes m_{1}$, the group $G$ acts by $\mathbb{k}$-linear transformations on $M$ via

$$
g . m=\sum m_{0} m_{1}(g) \quad(g \in G, m \in M) .
$$

Such $G$-actions, called rational $G$-actions, are in particular locally finite: the $G$-orbit of any $m \in M$ is contained in the finite-dimensional $\mathbb{k}$-subspace of $M$ that is generated by $\left\{m_{0}\right\}$. If $G$ acts rationally on $M$ then it does so on all $G$-subquotients of $M$. Moreover, every irreducible $G$-submodule of $M$ is finite dimensional, and the sum of all irreducible $G$-submodules is an essential $G$-submodule of $M$; it is called the socle of $M$ and denoted by $\operatorname{soc}_{G} M$. In the following, we will denote the set of isomorphism classes of irreducible $G$-modules by $\operatorname{irr} G$ and, for each $E \in \operatorname{irr} G$, we let

$$
[M: E] \in \mathbb{Z}_{\geq 0} \cup\{\infty\}
$$

denote the multiplicity of $E$ as a composition factor of $M$; see [13, I.2.14].

We will be primarily concerned with the situation where $G$ acts rationally by algebra automorphisms on a $\mathbb{k}$-algebra $R$. This is equivalent to $R$ being a right $\mathbb{k}[G]$-comodule algebra in the sense of $[\mathbf{2 6}, 4.1 .2]$. In the special case where $G \cong\left(\mathbb{k}^{\times}\right)^{d}$ is an algebraic torus, we have $\operatorname{irr} G=X(G) \cong \mathbb{Z}^{d}$, the lattice of rational characters $\lambda: G \rightarrow \mathbb{k}^{\times}$. We will usually write $\Lambda=X(G)$. A rational $G$-action on $R$ is equivalent to a $\mathbb{Z}^{d}$-grading $R=\bigoplus_{\lambda \in \mathbb{Z}^{d}} R_{\lambda}$ of the algebra $R$. This follows from the fact that $\mathbb{k}[G]$ is the group algebra $\mathbb{k} \Lambda$ of the lattice $\Lambda \cong \mathbb{Z}^{d}$, and $\mathbb{k} \Lambda$-comodule algebras are the same as $\Lambda$ graded algebras; see [26, 4.1.7]. The homogeneous component of $R$ of degree $\lambda$ is the 
weight space

$$
R_{\lambda}=\{r \in R \mid g . r=\lambda(g) r \text { for all } g \in G\},
$$

and $[R: \lambda]=\operatorname{dim}_{\mathbb{k}} R_{\lambda}$.

The following lemma was referred to in the Introduction.

Lemma 4. Let $R$ be a $\mathbb{k}$-algebra, where $\mathbb{k}$ is an algebraically closed field, and let $G$ be an affine algebraic $\mathbb{k}$-group that acts rationally by $\mathbb{k}$-algebra automorphisms on $R$. If $G^{0} \subseteq G$ denotes the connected component of the identity, then $G^{0}-\operatorname{Spec} R$ consists of all ordinary prime ideals of $R$ that are $G^{0}$-stable. Moreover, $G$-Spec $R$ is finite if and only if $G^{0}$-Spec is finite.

Proof. For the assertion that all $G^{0}$-primes are prime, see [21, Proposition 19(a)].

The second assertion, that $G$-Spec $R$ is finite if and only if $G^{0}$-Spec is so, actually holds for any (normal) subgroup $N \unlhd G$ having finite index in $G$ in place of $G^{0}$. Putting $\mathcal{G}=G / N$, we first note that the $G$-primes of $R$ are exactly the ideals of the form $P=\bigcap_{x \in \mathcal{G}} x . Q$ with $Q \in N$-Spec $R$. Indeed, $\bigcap_{x \in \mathcal{G}} x . Q$ is easily seen to be $G$ prime. Conversely, any $P \in G$-Spec $R$ has the form $P=P^{\prime}: G$ with $P^{\prime} \in \operatorname{Spec} R$ by [21, Proposition 8], and hence we may take $Q=P^{\prime}: N$. Moreover, the intersection $\bigcap_{x \in \mathcal{G}} x . Q$ determines the $N$-prime $Q$ to within $\mathcal{G}$-conjugacy, because all $x . Q$ are $N$-prime ideals of $R$ and $\mathcal{G}$ is finite. Therefore, finiteness of $N-\operatorname{Spec} R$ is equivalent to finiteness of $G-\operatorname{Spec} R$.

2.3. Some ring theoretic background on affine PI-algebras. Let $R$ be an affine PI-algebra over a commutative noetherian ring $\mathbb{k}$. The following facts are well known.

2.3.1. Semiprime ideals. The ring $R$ satisfies the ascending chain condition for semiprime ideals and, for each ideal $I$ of $R$, there are only finitely many primes of $R$ that are minimal over $I$. If $I$ is semiprime then $R / I$ is a right and left Goldie ring and the extended centroid of $R / I$, in the sense of Martindale [24], is given by $\mathcal{C}(R / I)=\mathcal{Z}(Q(R / I))$, the centre of the classical ring of quotients of $R / I$. If $I$ is prime, then $\mathcal{C}(R / I)$ is identical to the field of fractions of $\mathcal{Z}(R / I)$ by Posner's theorem. See $\left[\mathbf{3 0}, 6.1 .30,6.3 .36^{\prime}\right],[25,13.6 .9],[21,1.4 .2]$ for all this.

2.3.2. $G$-prime ideals. By Braun's theorem [30, 6.3.39], every ideal $I$ of $R$ contains a finite product of primes that contain $I$. As in the proof of Lemma 3, it follows that for any group $G$ acting by ring automorphisms on $R$, the $G$-primes of $R$ are exactly the ideals of the form $P: G$ with $P \in \operatorname{Spec} R$. Moreover, $P$ can be chosen to have a finite $G$-orbit. In particular, every $I \in G$-Spec $R$ is semiprime. The ring of $G$-invariants $\mathcal{C}(R / I)^{G}$ is a field for every $I \in G$-Spec $R$; see [21, Proposition 9].

2.3.3. Nullstellensatz. If $\mathbb{k}$ is a Jacobson ring then so is $R$ : every prime ideal of $R$ is an intersection of primitive ideals. Moreover, if $P$ is a primitive ideal of $R$ then $P$ is maximal; in fact, $\mathbb{k} / P \cap \mathbb{k}$ is a field and $R / P$ is a finite-dimensional algebra over this field; see [30, 6.3.3]. 
2.3.4. Rational ideals and the Dixmier-Meglin equivalence. Now assume that $\mathbb{k}$ is an algebraically closed field. Recall that a prime ideal $P$ of $R$ is said to be rational if $\mathcal{C}(R / P)=\mathbb{k}$ or, equivalently, $\mathcal{Z}(R / P)=\mathbb{k}$. By Posner's theorem [30, 6.1.30], this forces $P$ to have finite $\mathbb{k}$-codimension in $R$. In fact, for any prime ideal of $R$, the following properties coincide (Dixmier-Moglin equivalence), the implications $\Rightarrow$ being either trivial or immediate from the Nullstellensatz:

finite codimensional $\equiv$ maximal $\equiv$ locally closed in $\operatorname{Spec} R \equiv$ primitive $\equiv$ rational

2.4. The trace ring of a prime PI-ring. Let $R$ be a prime PI-ring with centre $C=\mathcal{Z}(R)$. By Posner's theorem [30, 6.1.30], the central localization $Q(R)=R_{C \backslash\{0\}}$ is a central simple algebra over the field of fractions $F=Q(C)=\mathcal{C}(R)$. For each $q \in Q(R)$ we can consider the reduced characteristic polynomial $c_{q}(X) \in F[X]$. In detail, letting $F^{\text {alg }}$ denote an algebraic closure of $F$, we have an isomorphism of $F^{\text {alg }}$-algebras

$$
\varphi: Q(R) \otimes_{F} F^{\mathrm{alg}} \cong \mathrm{M}_{n}\left(F^{\mathrm{alg}}\right)
$$

for some $n$. This isomorphism allows us to define $c_{q}(X)$ as the characteristic polynomial of the matrix $\varphi(q \otimes 1) \in \mathrm{M}_{n}\left(F^{\mathrm{alg}}\right)$. One can show that $c_{q}(X)$ has coefficients in $F$ and is independent of the choice of the isomorphism $\varphi$; see [28, Section 9a] or [6, Section 12.3].

The commutative trace ring of $R$, by definition, is the $C$-subalgebra of $F$ that is generated by the coefficients of all polynomials $c_{r}(X)$ with $r \in R$; this algebra will be denoted by $T$. The trace ring of $R$, denoted by $T R$, is the $C$-subalgebra of $Q(R)$ that is generated by $R$ and $T$. The following result is standard; see [25, 13.9.11] or [31, 3.2].

LEMMA 5. Let $R$ be a prime PI-ring that is an affine algebra over some commutative noetherian ring $\mathbb{k}$. Then $T$ is an affine commutative $\mathbb{k}$-algebra and $T R$ is a finitely generated $T$-module. Furthermore, $T R$ is finitely generated as $R$-module if and only if $R$ is noetherian.

Now suppose that a group $G$ acts by ring automorphisms on $R$. The action of $G$ extends uniquely to an action on the trace ring $T R$, and this action stabilizes $T$. To see this, note that the $G$-action on $R$ extends uniquely to an action on the ring of fractions $Q(R)$. Each $g \in G$ stabilizes $F=\mathcal{Z}(Q(R))$, and hence $g$ yields an automorphism of $F[X]$ via its action on the coefficients of polynomials. The reduced characteristic polynomials of $q \in Q(R)$ and of $g . q$ are related by

$$
c_{g . q}(X)=g \cdot c_{q}(X)
$$

Indeed, extending $g$ to a field automorphism of $F^{\text {alg }}$, we obtain automorphisms $\mathrm{M}_{n}(g) \in$ Aut $\mathrm{M}_{n}\left(F^{\mathrm{alg}}\right)$ and $\alpha_{g} \in$ Aut $Q(R) \otimes_{F} F^{\text {alg }}$, the latter being given by $\alpha_{g}(q \otimes f)=$ $g . q \otimes g . f$. Fixing $\varphi$ as in (1) we obtain an isomorphism of $F^{\text {alg }}$-algebras $\mathbf{M}_{n}(g)^{-1} \circ$ $\varphi \circ \alpha_{g}: Q(R) \otimes_{F} F^{\mathrm{alg}} \cong \mathrm{M}_{n}\left(F^{\mathrm{alg}}\right)$. Using this isomorphism to compute reduced characteristic polynomials, we see that $c_{q}(X)=g^{-1} \cdot c_{g . q}(X)$, proving (2). Since $g . r \in R$ for $r \in R$, equation (2) shows that the commutative trace ring $T$ is stable under the action of $G$ on $Q(R)$, and hence so is the trace ring $T R$. For rational actions, we have the following result of Vonessen [31, Proposition 3.4]. 
LemMa 6 (Vonessen [31]). Let $R$ a prime PI-algebra over an algebraically closed field $\mathbb{k}$ and let $G$ be an affine algebraic $\mathbb{k}$-group that acts rationally by $\mathbb{k}$-algebra automorphisms on $R$. Then the induced $G$-actions on TR and on $T$ are rational as well.

In general, the finiteness problem stated in the Introduction transfers nicely to trace rings.

Proposition 7. Let $R$ be a prime PI-ring that is an affine algebra over some commutative noetherian ring. Let $G$ be a group acting by ring automorphism on $R$ and consider the induced $G$-actions on $T$ and on $T R$. Then $G$-Spec $T$ is finite if and only if $G$-Spec $T R$ is finite. If $R$ is noetherian, then this is also equivalent to $G$-Spec $R$ being finite.

Proof. Lemma 3, applied to the finite centralizing extension $T \subseteq T R$ (Lemma 5), tells us that finiteness of $G$-Spec $T R$ is equivalent to finiteness of $G$-Spec $T$. If $R$ is noetherian, then we may argue in the same way for the finite centralizing extension $R \subseteq T R$.

3. Main result. Throughout this section, $R$ denotes an affine PI-algebra over an algebraically closed field $\mathbb{k}$ and $G$ will be an affine algebraic $\mathbb{k}$-group that acts rationally by $\mathbb{k}$-algebra automorphisms on $R$.

3.1. Sufficient criteria for $G$-rationality. By Proposition 1 we know that $G$-Spec $R$ is finite if and only if all $G$-primes of $R$ are $G$-rational. Therefore, $G$-rationality criteria are essential. As usual, the algebra $R$ will be called $G$-prime if the zero ideal of $R$ is $G$-prime; similarly for $G$-rationality.

Lemma 8. Assume that $R$ is G-prime.

(a) If there is an $N \in \mathbb{Z}$ such that $\left[\operatorname{soc}_{G} \mathcal{Z}(R): E\right] \leq N$ for all $E \in \operatorname{irr} G$ then $R$ is G-rational.

(b) If $G$ is connected solvable then $R$ is $G$-rational if and only if $\left[\operatorname{soc}_{G} \mathcal{Z}(R): E\right] \leq 1$ for all $E \in \operatorname{irr} G$.

Proof. (a) For a given $q \in \mathcal{C}(R)^{G}$ put $I=\{r \in R \mid q r \in R\}$; this is a nonzero $G$ stable ideal of $R$. Therefore, $J=I^{N} \cap \mathcal{Z}(R)$ is a nonzero $G$-stable ideal of $\mathcal{Z}(R)$; see [30, 6.1.28]. Note that $q^{i} J \subseteq \mathcal{Z}(R)$ for $0 \leq i \leq N$. We have $E \hookrightarrow J$ for some $E \in \operatorname{irr} G$ and multiplication with $q^{i}$ yields a $G$-equivariant map $E \hookrightarrow J \rightarrow \mathcal{Z}(R)$. Since $\operatorname{dim}_{\mathbb{k}} \operatorname{Hom}_{G}(E, \mathcal{Z}(R))=\left[\operatorname{soc}_{G} \mathcal{Z}(R): E\right] \leq N$, there are $k_{i} \in \mathbb{k}$, not all 0 , such that $c=\sum_{i=0}^{N} k_{i} q^{i}$ annihilates $E$. But nonzero elements of $\mathcal{C}(R)^{G}$ are invertible; so we must have $c=0$. Thus, $q$ is algebraic over $\mathbb{k}$ and so $q \in \mathbb{k}$.

(b) The condition is sufficient by part (a). For the converse, assume that $E_{1} \oplus E_{2} \subseteq$ $\mathcal{Z}(R)$ for isomorphic $E_{i} \in \operatorname{irr} G$. By the Lie-Kolchin Theorem [5, III.10.5], $E_{i}=\mathbb{k} x_{i}$ for suitable $x_{i}$. Since $x_{i}$ generates a $G$-stable two-sided ideal, $x_{i}$ is regular in $R$. The quotient $x_{1} x_{2}^{-1} \in \mathcal{C}(R)$ is a nonscalar $G$-invariant; so $R$ is not $G$-rational.

REMARK. A simplified version of the argument in the proof of (a), without recourse to [30, 6.1.28], establishes the following general fact: Let $A$ be an arbitrary (associative) $\mathbb{k}$-algebra and let $G$ be a group that acts on $A$ by locally finite $\mathbb{k}$-algebra automorphisms. If there is an $N \in \mathbb{Z}$ such that $[A: E] \leq N$ holds for all finite-dimensional irreducible 
$\mathbb{k} G$-modules $E$, where $[A: E]$ denotes the multiplicity of $E$ as composition factor of $A$ as in [13, I.2.14], then $G$-Spec $A=G$-Rat $A$.

3.2. Regular primes. Recall from (1) that if $R$ is prime, then the classical ring of quotients $Q(R)$ is a central simple algebra over the field of fractions $F=Q(\mathcal{Z}(R))$. The $P I$ degree of $R$, by definition, is the degree of this central simple algebra: PI $\operatorname{deg} R=$ $\sqrt{\operatorname{dim}_{F} Q(R)}$. For any $P \in \operatorname{Spec} R$, one has PIdeg $R / P \leq \operatorname{PI} \operatorname{deg} R$. The prime $P$ is called regular if equality holds here. The regular primes form an open subset of $\operatorname{Spec} R$. See [30, p. 104] or [25, 13.7.2] for all this.

Now let $G$ be an algebraic $\mathbb{k}$-torus. In particular, $G$ is connected and so $G$-Spec $R$ consists of the $G$-stable prime ideals of $R$ by Lemma 4 .

LEMmA 9. Let $G$ be an algebraic $\mathbb{k}$-torus and assume that $R$ is prime. Then, for every regular $P \in G$-Spec $R$, we have $\operatorname{tr} \operatorname{deg}_{\mathbb{k}} \mathcal{C}(R / P)^{G} \leq \operatorname{tr} \operatorname{deg}_{\mathbb{k}} \mathcal{C}(R)^{G}$. Consequently, if $R$ is $G$-rational then all regular primes in $G$-Spec $R$ belong to $G$-Rat $R$.

Proof. Let $P \in G$-Spec $R$ be regular. Put $n=$ PIdeg $R$ and let $g_{n}(R)^{+}$denote the Formanek centre of $R$; this is a $G$-stable ideal of $\mathcal{Z}(R)$ such that $g_{n}(R)^{+} \not \subset P$ (cf. [30, 6.1.37] or [25, 13.7.2(i)]). Therefore, we may choose a semi-invariant $c \in g_{n}(R)_{\lambda}^{+}$with $c \notin P$. The group $G$ acts rationally on the localization $R_{c}=R[1 / c]$ and $R_{c}$ is Azumaya by the Artin-Procesi theorem [25, 13.7.14]. Therefore, $\mathcal{Z}\left(R_{c}\right)$ maps onto $\mathcal{Z}\left(R_{c} / P R_{c}\right)$ and $\mathcal{Z}\left(R_{c}\right)_{\lambda}$ maps onto $\mathcal{Z}\left(R_{c} / P R_{c}\right)_{\lambda}$ for all $\lambda \in X(G)$. The map $\mathcal{Z}\left(R_{c}\right) \rightarrow \mathcal{Z}\left(R_{c} / P R_{c}\right)$ extends to a $G$-equivariant epimorphism $\mathcal{Z}\left(R_{\mathfrak{p}}\right) \rightarrow \mathcal{C}(R / P)=Q\left(\mathcal{Z}\left(R_{c} / P R_{c}\right)\right)$, where $\mathfrak{p}=P \cap \mathcal{Z}(R)$. Since $\mathcal{Z}\left(R_{\mathfrak{p}}\right)^{G} \subseteq \mathcal{C}(R)^{G}$, it suffices to show that $\mathcal{Z}\left(R_{\mathfrak{p}}\right)^{G}$ maps onto $\mathcal{C}(R / P)^{G}$. But, given $q \in \mathcal{C}(R / P)^{G}$, we can find a semi-invariant $0 \neq x \in \mathcal{Z}\left(R_{c} / P R_{c}\right)_{\lambda}$ such that $q x \in \mathcal{Z}\left(R_{c} / P R_{c}\right)$, and we can further find $y, z \in \mathcal{Z}\left(R_{c}\right)_{\lambda}$ with $y \mapsto x$ and $z \mapsto q x$. Then $z y^{-1} \in \mathcal{Z}\left(R_{\mathfrak{p}}\right)^{G}$ maps to $q$. This proves the lemma.

3.3. Proof of Theorem 2. Let $G$ be an algebraic $\mathbb{k}$-torus and assume that $R$ is prime. We need to show that $G$-Spec $R$ is finite if and only if the action of $G$ on $\mathcal{Z}(R)$ is multiplicity free. By Lemma $8(\mathrm{~b})$, the latter property is equivalent to $G$-rationality of $R$, and this is certainly necessary for $G-\operatorname{Spec} R$ to be finite by Proposition 1 .

Now assume that $R$ is $G$-rational. By Proposition 1 we must show that all $G$-primes of $R$ are $G$-rational. Lemma 9 ensures this for the regular $G$-primes. In particular, we may assume that $n:=\mathrm{PI} \operatorname{deg} R>1$. Now consider $P \in G$-Spec $R$ with PI $\operatorname{deg} R / P<n$. Then $P$ contains the ideal $\mathfrak{a}=g_{n}(R) R \subseteq R$; this is a nonzero $G$-stable common ideal of $R$ and of the trace ring $R^{\prime}:=T R$ of $R$ (cf. [30, 6.1.37 and 6.3.28]). All primes of $R$ that are minimal over $\mathfrak{a}$ are $G$-stable. Let $Q$ be one of these primes such that $Q \subseteq P$. It suffices to show that $Q$ is $G$-rational. For, then we may replace $R$ by $R / Q$, and since PI $\operatorname{deg} R / Q<n$, we may argue by induction that $P$ is $G$-rational.

First, we claim that there exists $Q^{\prime} \in G$-Spec $R^{\prime}$ with $Q^{\prime} \cap R=Q$. Indeed, choosing $Q^{\prime}$ to be a $G$-stable ideal of $R^{\prime}$ that is maximal subject to the condition $Q^{\prime} \cap R \subseteq Q$, it is straightforward to see that $Q^{\prime}$ is $G$-prime. If $Q^{\prime} \cap R \neq Q$ then $Q^{\prime} \nsupseteq \mathfrak{a}$ by minimality of $Q$ over $\mathfrak{a}$. Thus, $Q^{\prime}+\mathfrak{a}$ is a $G$-stable ideal of $R^{\prime}$ which properly contains $Q^{\prime}$ and yet also satisfies $\left(Q^{\prime}+\mathfrak{a}\right) \cap R=\left(Q^{\prime} \cap R\right)+\mathfrak{a} \subseteq Q$. Since this contradicts our maximal choice of $Q^{\prime}$, we must have $Q^{\prime} \cap R=Q$ as claimed.

Next, we show that $Q^{\prime}$ is $G$-rational. To see this, recall from Lemma 6 that $G$ acts rationally on the trace rings $T$ and $R^{\prime}$. Moreover, $T$ is an affine commutative $\mathbb{k}$-algebra that is $G$-rational, because $Q(T)^{G}=\mathcal{C}(R)^{G}=\mathbb{k}$. Therefore, by the case $n=1$, we know 
that $G$-Spec $T$ is finite. By Proposition 7, $G$-Spec $R^{\prime}$ is finite as well, and in view of Proposition 1, this forces $Q^{\prime}$ to be $G$-rational.

Finally, we show that $Q$ is $G$-rational; this will finish the proof. But $\mathcal{C}(R / Q) \subseteq$ $\mathcal{C}\left(R^{\prime} / Q^{\prime}\right)$ and $\mathcal{C}\left(R^{\prime} / Q^{\prime}\right)^{G}=\mathbb{k}$ by the foregoing. Therefore, $\mathcal{C}(R / Q)^{G}=\mathbb{k}$ as desired.

4. Related results. In this section, $R$ and $G$ are as in the previous section and $R$ is also assumed noetherian.

4.1. Actions of reductive groups. Recall from Lemma 6 that the induced $G$-action on the commutative trace ring $T$ is rational. This fact allows us to invoke results from algebraic geometry.

Proposition 10. Let $R$ be an affine noetherian PI-algebra over an algebraically closed field $\mathbb{k}$ and let $G$ be an affine algebraic $\mathbb{k}$-group that acts rationally by $\mathbb{k}$-algebra automorphisms on $R$. Assume that $R$ is prime and that $G$ is connected reductive. Let $F=Q(\mathcal{Z}(R))$ denote the field of fractions of the centre of $R$, and let $F^{B} \subseteq F$ denote the invariant subfield of a Borel subgroup $B \leq G$. If $F^{B}=\mathbb{k}$ then $B$-Spec $R$ is finite (and hence $G$-Spec $R$ is finite as well).

Proof. By Proposition 7, $B$-Spec $R$ is finite if and only if $B$-Spec $T$ is finite. Now, $T$ is an affine commutative domain over $\mathbb{k}$ and the field of fractions of $T$ is $F$. By a standard result on spherical varieties [16, Corollary 2.6], the condition $F^{B}=\mathbb{k}$ implies that there are only finitely many $B$-orbits in Rat $T$. The latter fact is equivalent to finiteness of $B$-Spec $T$ by Proposition 1, which proves the proposition.

4.2. Catenarity. A partially ordered $\operatorname{set}(P, \leq)$ is said to be catenary if, given any two $x<x^{\prime}$ in $P$, all saturated chains $x=x_{0}<x_{1}<\cdots<x_{r}=x^{\prime}$ have the same finite length $r=r\left(x, x^{\prime}\right)$.

In the commutative case, the following observation goes back to conversations that I had with R. Rentschler a long time ago; cf. [23, Section 3]. As usual, GK dim denotes Gelfand-Kirillov dimension.

Proposition 11. Let $R$ be an affine noetherian PI-algebra over an algebraically closed field $\mathbb{k}$ and let $G$ be an affine algebraic $\mathbb{k}$-group that acts rationally by $\mathbb{k}$-algebra automorphisms on $R$. If the connected component of the identity of $G$ is solvable then the poset $(G$-Spec $R, \subseteq)$ is catenary. In fact, every saturated chain $P=P_{0} \subset P_{1} \subset \cdots \subset$ $P_{r}=P^{\prime}$ in $G$-Spec $R$ has length $r=\mathrm{GK} \operatorname{dim} R / P-\mathrm{GK} \operatorname{dim} R / P^{\prime}$.

Proof. First assume that $G$ is connected; so $G$-Spec $R$ consists of the $G$-stable primes of $R$. In view of Schelter's catenarity theorem for $\operatorname{Spec} R$ [30,6.3.43], we need to show that any two neighbours $Q \subset P$ in $G$-Spec $R$ are also neighbours when viewed in Spec $R$. Passing to $R / Q$ we may assume that the algebra $R$ is prime and $P$ is a minimal nonzero member of $G$-Spec $R$, and we need to show that $P$ has height 1 in $\operatorname{Spec} R$. But $P \cap \mathcal{Z}(R)$ is a nonzero $G$-stable ideal of $\mathcal{Z}(R)$ and hence the Lie-Kolchin theorem provides us with a $G$-eigenvector $0 \neq z \in P \cap \mathcal{Z}(R)$. The ideal $P$ is a minimal prime over (z). For, if $(z) \subseteq P^{\prime} \subset P$ for some $P^{\prime} \in \operatorname{Spec} R$ then $(z) \subseteq P^{\prime}: G \subset P$ and $P^{\prime}: G \in G$-Spec $R$, contradicting the fact that $P$ is a minimal nonzero member of $G$-Spec $R$. Thus, $P$ is minimal over $(z)$ as claimed, and the principal ideal theorem $[\mathbf{2 5}, 4.1 .11]$ gives that $P$ has height 1 as desired. 
In general, let $G^{0}$ denote the connected component of the identity of $G$ and put $\mathcal{G}=G / G^{0}$. Write $Q=\bigcap_{x \in \mathcal{G}} x \cdot \widetilde{Q}$ and $P=\bigcap_{x \in \mathcal{G}} x . \widetilde{P}$ for suitable $\widetilde{Q}, \widetilde{P} \in G^{0}$-Spec $R$ as in the proof of Lemma 4. Since these intersections are finite intersections of $G^{0}$ primes of $R$, we can arrange that $\widetilde{Q} \subset \widetilde{P}$. The ideals $\widetilde{Q}$ and $\widetilde{P}$ are neighbours in $G^{0}$-Spec $R$. For, if $\widetilde{Q} \subset \widetilde{T} \subset \widetilde{P}$ for some $\widetilde{T} \in G^{0}-\operatorname{Spec} R$ then $Q \subset \bigcap_{x \in \mathcal{G}} x . \widetilde{T} \subset P$ since $\mathcal{G}$ is finite, which contradicts the fact that $Q$ and $P$ are neighbours in $G$-Spec $R$. By the first paragraph of the proof, $\widetilde{Q}$ and $\widetilde{P}$ are also neighbours in Spec $R$, and hence $\mathrm{GK} \operatorname{dim} R / \widetilde{Q}=\mathrm{GK} \operatorname{dim} R / \widetilde{P}+1$ by Schelter's theorem. Since GK $\operatorname{dim} R / Q=$ $\mathrm{GK} \operatorname{dim} R / \widetilde{Q}$ and $\mathrm{GK} \operatorname{dim} R / P=\mathrm{GK} \operatorname{dim} R / \widetilde{P}$ by [18, Corollary 3.3], the proof is complete.

ACKNOWLEDGEMENTS. A preliminary version of some of these results was presented during the CIMPA school 'Braids in Algebra, Geometry and Topology' in Hanoi (17-28 January 2011). The author thanks Ken Goodearl, Friedrich Knop, Claudio Procesi and the referee for valuable comments and suggestions. This research was supported in part by NSA grant H98230-09-1-0026.

\section{REFERENCES}

1. G. Abrams, J. P. Bell and K. M. Rangaswamy, The Dixmier-Moeglin equivalence for Leavitt path algebras Algebr. Represent. Theory 15(3) (2012), 407-425.

2. S. A. Amitsur, The identities of PI-rings, Proc. Amer. Math. Soc. 4 (1953), 27-34.

3. G. M. Bergman, Lifting prime ideals to extensions by centralizing elements, unpublished, University of California, Berkeley.

4. G. M. Bergman, More on extensions by centralizing elements, unpublished, University of California, Berkeley.

5. A. Borel, Linear algebraic groups, 2nd ed., Graduate Texts in Mathematics, vol. 126 (Springer-Verlag, New York, 1991).

6. N. Bourbaki, Éléments de mathématique, Fasc. XXIII, Hermann, Paris, 1973, Livre II: Algèbre. Chapitre 8: Modules et anneaux semi-simples, Nouveau tirage de l'édition de 1958, Actualités Scientifiques et Industrielles, No. 1261.

7. K. A. Brown and K. R. Goodearl, Lectures on algebraic quantum groups, Advanced Courses in Mathematics - CRM Barcelona (Birkhäuser Verlag, Basel, 2002).

8. G. Cauchon, Effacement des dérivations et spectres premiers des algèbres quantiques, J. Algebra 260(2) (2003), 476-518.

9. G. Cauchon, Spectre premier de $O_{q}\left(M_{n}(k)\right)$ : image canonique et séparation normale, J. Algebra 260(2) (2003), 519-569.

10. K. R. Goodearl and E. S. Letzter, Prime ideals in skew and $q$-skew polynomial rings, Mem. Amer. Math. Soc. 109(521) (1994), vi+106.

11. K. R. Goodearl and E. S. Letzter, The Dixmier-Moeglin equivalence in quantum coordinate rings and quantized Weyl algebras, Trans. Amer. Math. Soc. 352(3) (2000), 13811403.

12. T. J. Hodges, T. Levasseur and $M$. Toro, Algebraic structure of multiparameter quantum groups, Adv. Math. 126(1) (1997), 52-92.

13. J. C. Jantzen, Representations of algebraic groups, 2nd ed., Mathematical Surveys and Monographs, vol. 107 (American Mathematical Society, Providence, RI, 2003).

14. A. Joseph, Quantum groups and their primitive ideals, Ergebnisse der Mathematik und ihrer Grenzgebiete (3) [Results in Mathematics and Related Areas (3)], vol. 29, (Springer-Verlag, Berlin, 1995).

16. F. Knop, On the set of orbits for a Borel subgroup, Comment. Math. Helv. 70(2) (1995), 285-309. 
17. H. Kraft, Geometrische Methoden in der Invariantentheorie, Aspects of Mathematics, D1 (Friedr. Vieweg \& Sohn, Braunschweig, 1984).

18. G. R. Krause and T. H. Lenagan, Growth of algebras and Gelfand-Kirillov dimension, revised ed., Graduate Studies in Mathematics, vol. 22 (American Mathematical Society, Providence, RI, 2000).

19. S. Launois, T. H. Lenagan and L. Rigal, Prime ideals in the quantum Grassmannian, Selecta Math. (N.S.) 13(4) (2008), 697-725.

20. M. Lorenz, Finite normalizing extensions of rings, Math. Z. 176(4) (1981), 447-484.

21. M. Lorenz, Group actions and rational ideals, Algebra Number Theory 2(4) (2008), 467-499.

22. M. Lorenz, Algebraic group actions on noncommutative spectra, Transform. Groups 14(3) (2009), 649-675.

23. M.-P. Malliavin, Caténarité et théorème d'intersection en algèbre non commutative, Séminaire d'Algèbre Paul Dubreil 31ème année (Paris, 1977-1978), Lecture Notes in Mathematics, vol. 740 (Springer, Berlin, 1979), 408-431.

24. W. S. Martindale, III, Prime rings satisfying a generalized polynomial identity, J. Algebra 12 (1969), 576-584. MR MR0238897 (39 \#257)

25. J. C. McConnell and J. C. Robson, Noncommutative Noetherian rings, revised ed., Graduate Studies in Mathematics, vol. 30 (American Mathematical Society, Providence, RI, 2001), With the cooperation of L. W. Small.

26. S. Montgomery, Hopf algebras and their actions on rings, CBMS Regional Conference Series in Mathematics, vol. 82 (CBMS, Washington, DC, 1993).

27. C. Procesi, Rings with polynomial identities Pure and Applied Mathematics, vol. 17 (Marcel Dekker Inc., New York, 1973 ).

28. I. Reiner, Maximal orders, London Mathematical Society Monographs, New Series, vol. 28 (The Clarendon Press, Oxford University Press, Oxford, 2003); corrected reprint of the 1975 original, with a foreword by M. J. Taylor.

29. J. C. Robson and L. W. Small, Liberal extensions, Proc. London Math. Soc. (3) 42(1) (1981), 87-103.

30. L. H. Rowen, Ring theory, Vol. II, Pure and Applied Mathematics, vol. 128 (Academic Press, Boston, MA, 1988).

31. N. Vonessen, Actions of linearly reductive groups on affine PI algebras, Mem. Amer. Math. Soc. 81(414) (1989), iv+106.

32. W. C. Waterhouse, Introduction to affine group schemes, Graduate Texts in Mathematics, vol. 66 (Springer-Verlag, New York, 1979). 